\title{
Exploring Polysaccharide lyases of PL-5 family through the lens of structure, function, and dynamics
}

\author{
Prerana Dash ${ }^{1,2}$, Rudresh Acharya ${ }^{1,2}$ \\ ${ }^{I}$ National Institution of science education and research, Bhubaneswar, India; \\ ${ }^{2}$ Homi Bhabha National Institute, Training School Complex, Anushakti Nagar, Mumbai 400094, Maharashtra, India \\ prerana.dash@niser.ac.in
}

Polysaccharide lyases are the biologically and industrially important enzymes, which catalyze non-hydrolytic degradation of polysaccharides via a beta-elimination reaction mechanism. There are 40 families of PLs in the CAZY database; classified based on their secondary structure elements and the folds. The PL-5 family enzyme adopts (alpha/alpha) $)_{5,5}$ fold with an N-terminal lid-loop interaction giving rise to a pseudo-toroid architecture. Our research group work is focused on delineating the structure-functiondynamics for PL-5 family enzymes. To this end, the biochemical characterization has been carried on the selected among PL-5 enzymes to identify substrate specificity and enzyme efficiency. We have determined the X-ray crystal structures of the enzymes in apo and substrate-bound forms to understand structural aspects of substrate acquisition and specificity as a function of $\mathrm{pH}$ and the enzyme-substrate interactions. Further, the molecular dynamic simulation performed on the X-ray structures suggest the potential dynamics in loop configuration of the molecule to a closed and open state; providing mechanistic insights into functioning, and the mechanism of substrate acquisition and product expulsion in the PL-5 family enzymes.

Keywords: beta-elimination, pseudo-toroid, Molecular dynamic simulation, substrate aquisition 